\title{
Corela
}

Cognition, représentation, langage

HS-33 | 2020

Textuel, textiel. Repenser la textualité numérique

\section{Quand le discours de savoir se fait technodiscours. Hypertextualité, commentaires et unité textuelle du billet scientifique}

Ingrid Mayeur

\section{(2) OpenEdition}

Journals

Édition électronique

URL : https://journals.openedition.org/corela/11876

DOI : $10.4000 /$ corela.11876

ISSN : 1638-573X

Éditeur

Cercle linguistique du Centre et de l'Ouest - CerLICO

Référence électronique

Ingrid Mayeur, "Quand le discours de savoir se fait technodiscours. Hypertextualité, commentaires et unité textuelle du billet scientifique », Corela [En ligne], HS-33 | 2020, mis en ligne le 24 septembre 2020, consulté le 14 juillet 2021. URL : http://journals.openedition.org/corela/11876 ; DOI : https:// doi.org/10.4000/corela.11876

Ce document a été généré automatiquement le 14 juillet 2021.

\section{(c) (i) (3)}

Corela - cognition, représentation, langage est mis à disposition selon les termes de la licence Creative Commons Attribution - Pas d'Utilisation Commerciale - Partage dans les Mêmes Conditions 4.0 International. 


\title{
Quand le discours de savoir se fait technodiscours. Hypertextualité, commentaires et unité textuelle du billet scientifique ${ }^{1}$
}

\author{
Ingrid Mayeur
}

La communication scientifique écrite repose ordinairement sur des formes stabilisées, liées à une instance énonciative identifiable : en sciences humaines et sociales (SHS), l'article de revue ou la monographie sont ainsi les genres dominants. L'environnement numérique transforme ces genres de la recherche, par l'adjonction de métadonnées, la fragmentation et l'agrégation au sein de portails (Beaudry 2011), tandis qu'émergent de nouveaux modes de diffusion comme le blogging scientifique (mobilisant le cas échéant des imaginaires liés à des usages antérieurs, tel le carnet de recherche: cf. Dacos et Mounier 2010). Si les sciences de l'information et de la communication (SIC) ont porté l'attention sur la nature désormais opératoire du texte devenu textiel, associant les composantes langagières et techniques (Davallon et al. 2013) ainsi que sur les possibilités ouvertes par les pratiques d'écrilecture des textes scientifiques (Gallezot 2016 ; Kembellec et Broudoux 2017) - elles-mêmes inscrites dans le sillage de la lettrure médiévale, où la lecture des textes savants était déjà indissociable d'une action d'écriture (Souchier 2012) -, on ne semble pas encore disposer de critères à même de dégager ce qui fait texte dans les discours de savoir ${ }^{2}$ numériques, ou, plus précisément, ce qui permet au discours scientifique de s'énoncer en tant que forme cohérente, circulante et citable $^{3}$, tout en se présentant, $d u$ fait de ses propriétés technodiscursives ${ }^{4}$, comme un texte susceptible d'ajouts continus, manipulable et délinéarisable (Paveau 2016). Le technodiscours apparaît ainsi comme un composite, mêlant intimement, à l'instar du textiel dont il interroge le dispositif d'énonciation, les matières linguistiques et technologiques. Si, dans la perspective de Paveau, la notion procède des travaux de Latour sur l'assemblage (Latour 1991), elle a été théorisée dans une autre perspective par Le Marec afin de rendre compte des entrelacements entre pratiques de communication de la recherche et pratiques scientifiques (Le Marec 2002). 
Le propre d'un composite semble ainsi de compliquer la délimitation d'un dedans et d'un dehors, d'un intérieur et d'un extérieur : on comprend dès lors en quoi les énoncés composites des billets de recherche en ligne contribuent à questionner les processus de textualisation.

2 La production scientifique se construisant habituellement sur la reprise et l'évaluation des thèses antérieures, le discours de savoir numérique est traversé d'une tension entre les possibilités ouvertes par l'instabilité de ses frontières (entendues ici comme ce qui délimiterait un extérieur du texte) et la nécessité d'une unité textuelle stable permettant la citation en vue d'une mobilisation dans des travaux ultérieurs. Sur la base de ce constat, nous entendons mener ici une enquête exploratoire à partir d'un corpus de billets de recherche publiés sur Hypothèses, plateforme de blogging en SHS intégrée au portail OpenEdition, en faisant porter l'attention sur deux pratiques qui semblent a priori compliquer l'appréhension des billets comme unités textuelles de savoir : l'enrichissement hypertextuel ${ }^{5}$ et le commentaire. Nous espérons ainsi apporter quelques propositions pour identifier ce qui contribue à faire texte dans les discours scientifiques numériques des blogs de recherche.

\section{Quelle(s) unité(s) textuelle(s) dans l'environnement numérique?}

Enrichissement hypertextuel et commentaires manifestent des formes par lesquelles l'unité textuelle semble mise en crise; on pourrait sans doute, à cet égard, les rapprocher des figures d'ajout étudiées dans un ouvrage collectif du même nom dirigé par Authier-Revuz et Lala (Authier-Revuz et Lala 2002). Ces figures concernent plus largement ce qui est ressenti comme une extériorité par rapport à un texte jugé principal (digressions, notes, ajouts génétiques, etc.), ce qui fait ajout: ainsi que le signale Combe dans sa contribution, "[1]a question de fond pour l'ajout, est en définitive celle des frontières du texte, et par là de la textualité » (Combe 2002, 18) - ce qui fait directement écho au propos de Todorov, définissant le texte par son autonomie et sa clôture (Ducrot et Todorov 1972, 375). Cette problématique des figures d'ajout interroge donc les saillances du texte et établit une hiérarchie, un rapport dissymétrique entre les composantes d'un même texte. Le relief qui en résulte sert alors la dramatisation du discours : «La figure de l'ajout participe ainsi d'une mise en scène de la dynamique du dire, donnant corps à ses mouvements successifs » (Authier-Revuz et Lala 2002, 9). L'écriture numérique semble reposer, fondamentalement, sur une poétique de l'ajout (pouvant se manifester sous la forme de liens hypertextuels, d'annotations, d'étiquettes, etc.), au sein d'un processus continu : l'écriture se poursuit en effet, le cas échéant, après la mise en circulation originelle du texte, et résulte parfois de l'action d'un scripteur autre que celui qui en était à l'initiative ${ }^{6}$. À cet égard, si les technomots ou technosegments, associant un hyperlien et un élément linguistique (Paveau 2017), se situent du côté de l'ajout autographe, de la main du locuteur premier ${ }^{7}$, et, de ce fait, ressortissent davantage aux questions abordées dans le collectif d'Authier-Revuz et Lala en ce qu'ils font apparaître une altérité dans le discours de celui-ci (Authier-Revuz et Lala 2002, 11) ${ }^{8}$, le commentaire en revanche ressortirait plutôt à l'ajout allographe, émanant d'un locuteur second'. 


\section{Ce qui fait texte dans les écrits des blogs}

4 En réalité, l'approche menée dans le collectif dirigé par Authier-Revuz et Lala apparaît complémentaire à celle de la linguistique textuelle d'Adam : la problématique de ce qui fait ajout ne peut se penser qu'au regard de ce qui fait texte (Adam 2015), de la matérialité par rapport à laquelle cet ajout fait écart. Mais, une fois encore, l'appréhension d'un texte comme unité résulte d'un ressenti mobilisant, en production et en réception, la reconnaissance d'opérations de liage. Adam propose ainsi de substituer, à la question "Qu'est-ce qu'un texte ", une réflexion sur les facteurs qui amènent un sujet écrivant ou lisant à porter sur une suite d'énoncé un jugement de textualité et sur les conséquences de ce jugement sur l'interprétation des énoncés. Ce jugement se fonde sur un triple sentiment: de connexité (liages micro-textuel des énoncés), de cohésion (sentiment de totalité locale et globale, des parties elles-mêmes et des parties en relation avec le tout textuel) et de cohérence (sentiment d'adéquation des énoncés à une situation socio-discursive et à un genre de discours). (Adam 2015, $45-46)^{10}$

5 Comme technogenre prescrit (Paveau 2017, 301), l'écrit de blog correspondrait à un genre de discours suggéré ${ }^{11}$ par le dispositif d'écriture et fortement contraint par lui : on attend ainsi de ce dispositif médiatique qu'il livre des publications sérielles, organisées par ordre antéchronologique, qu'il autorise les commentaires, etc. ${ }^{12}$. S'il est nécessaire de nuancer l'appréhension du blog en tant que genre (voir par exemple les observations de Maingueneau 2013, qui préférerait y voir un hypergenre aux contraintes formelles minimales; ou de Candel 2010 qui soulignait la « vicariance » du blog, soit sa capacité à se plier aux fonctions communicationnelles les plus diverses), il n'en reste pas moins que les architextes informatisés dont il dépend agissent comme des outils d'écriture en amont du texte (Jeanneret et Souchier 2005), faisant intervenir d'autres énonciateurs (informaticiens, programmeurs, etc.). De l'architexte dépend ainsi la production matérielle des frontières textuelles de l'écrit de blog: déterminant les contours du texte en tant qu'unité susceptible de circuler dans des espaces externes à son lieu d'inscription premier - puisqu'en effet, "le texte naît de l'architexte qui en balise l'écriture » (Souchier et Jeanneret 1999, 103) -, il apparaît ainsi responsable de la production d'une forme-texte balisée, de cadres visuels, d'une énonciation éditoriale porteuse de sens dans une culture donnée et anticipant certains usages du lecteur étant donné que les savoirs, afin de circuler, nécessitent d'être formalisés en textes (Jeanneret 2005, 45' sqq.). Sur la plateforme Hypothèses, la production matérielle du billet est conditionnée par l'architexte WordPress; depuis son lieu de publication originel (le carnet), le billet comme forme circulante peut faire l'objet d'éditorialisations ${ }^{13}$ dans des espaces sociaux variés (p. ex. valorisation en page d'accueil d'Hypothèses, relais sur Facebook ou Twitter, etc.). Le sentiment de cohésion textuelle qui naît à ce niveau résulte de l'embarquement d'un ensemble de données et métadonnées marqué par la pluralité des instances énonciatives à l'œuvre dans la production du billet ; l'architexte manipulé par le scripteur pourrait, en quelque sorte, assurer l'acte macro-énonciatif qui ferait tenir ensemble les composantes du billet comme texte, tout en établissant une hiérarchie entre elles (à cet égard, les commentaires ou les étiquettes apparaissent comme secondaires par rapport au corps de texte). En effet, pour Adam, 
6 Deux types d'opérations font d'un texte un tout configuré : l'établissement d'une unité sémantique (thématique) globale et (au moins) d'un acte de discours dominant. Unité thématique et unité illocutoire déterminent la cohérence sémantico-pragmatique globale d'un texte (ou d'une partie de texte). (Adam 2011, 204)

$7 \mathrm{Au}$ demeurant, l'unité illocutoire résultant d'un tel acte macro-énonciatif pose question : celle-ci, comme la cohérence thématique, ne saurait être prise en charge par l'architexte mais reste tributaire du locuteur premier, dont l'activité discursive est toutefois régie par les contraintes d'écriture du dispositif informatisé.

8 La notion de jugement de textualité amène à impliquer le lecteur dans les opérations de textualisation $^{14}$; elle se fonde, notamment, sur des savoir-faire acquis par la fréquentation de la culture écrite, sur la littéracie de l'usager ${ }^{15}$. Cependant, dans l'environnement numérique plus encore qu'ailleurs, le texte apparait bien comme la résultante d'une négociation ${ }^{16}$ entre scripteur et lecteur; ce qu'avaient montré les recherches en SIC sur le textiel. Le texte numérique est pensé, dans cette perspective, au regard des usages que serait susceptible d'en faire le lecteur comme « acteur engagé dans un programme d'activité » (Davallon, Noël-Cadet, et Brochu 2013, § 112), impliqué dans la composition d'un « texte pouvant lui servir » (Ibid., § 96) : le lecteur se fait ainsi écrilecteur (Barbosa 1992; cité par Paveau 2016), dessinant les contours du texte (en personnalisant les paramètres d'affichage, en activant des liens, etc.) selon ses intérêts.

\section{Horizon de questionnement pour l'analyse du corpus}

Cette dimension nous ramène à la spécificité de notre objet, constitué de discours scientifiques publiés sur blog. Pour cerner l'extension du discours scientifique, on se ralliera à la définition de Rinck qui en fait un «discours produit dans le cadre de l'activité de recherche à des fins de construction et de diffusion du savoir.» (Rinck $2010, \S 2)$; comme plateforme de communication scientifique, Hypothèses diffuse des contenus pouvant se rattacher à cette acception assez large. Nous voudrions dès lors, d'une part, rendre compte du fonctionnement des hypertextes et des commentaires dans les billets de recherche mais aussi, d'autre part, interroger ce qui fait texte dans le discours scientifique de ces blogs au regard de ces formes et de leurs usages, en tant qu'elles participent d'un projet communicationnel qui vise la diffusion et l'appropriation des savoirs par une éventuelle remobilisation en pôle réception. Comment le billet de recherche négocie-t-il l'intégration, comme ajout autographe dans le cas de l'enrichissement hypertextuel, comme ajout allographe dans le cas du commentaire, à ce qui serait ressenti de prime abord comme un texte premier constitué du corps du billet (si l'on se réfère à la proposition de Todorov mentionnée plus haut, définissant le texte à la fois par son autonomie et sa clôture), de manière à entretenir un sentiment plus ou moins fort de cohésion, connexité, cohérence avec celui-ci ? Pour apporter des éléments de réponse à ces questions, on fonctionnera en deux temps : d'abord, en identifiant comment se manifestent ces deux formes dans les discours scientifiques d'Hypothèses et comment elles se greffent sur la trame du billet en étant susceptible de créer un jugement plus ou moins fort de textualité ; la conclusion proposera ensuite une lecture des écrits hypertextualisés et des commentaires comme gestes discursifs du savoir (Lttr13 2016), contribuant à accroître le dynamisme du discours scientifique numérique et, par là, à renforcer le jugement de textualité porté sur les billets. Notre analyse exploratoire reposera sur un corpus constitué de 87 billets de blog 
extraits de la Une de la page d'accueil d'Hypothèses dans sa section francophone, durant trois séquences temporelles (15/10/2016-15/01/2018, 15/04/2017-15/07/2017, $15 / 10 / 2017-15 / 01 / 2018)^{17}$.

\section{Le discours scientifique hypertextualisé ${ }^{18}$}

10 Les billets de blog, comme écrits numériqués (Paveau 2015) - soit, produits dans des architextes informatisés en réseau -, possèdent un degré de relationalité élevé en ce qu'ils sont originellement ancrés dans l'écosystème du web. Si, sur Hypothèses, les chercheurs-carnetiers usent diversement des possibilités de liage hypertextuel de leurs billets (certains, par exemple, ne mobilisent aucun technomot), le recours à l'hypertexte comme technologie discursive (Paveau 2016) s'associe à des enjeux de savoir inédits. En effet, manifestant typographiquement - à l'instar de la citation - la présence d'un texte dans un autre, l'enrichissement hypertextuel participe des phénomènes d'intertextualité, sans pour autant s'y réduire ${ }^{19}$. Toutefois, contrairement à la citation, il ne reproduit pas un extrait de discours autre lié à une instance énonciative, mais fournit les coordonnées d'une totalité textuelle ainsi qu'une possibilité de délinéarisation associée. Le technomot/technosegment permet ainsi, le cas échéant, la mise en relation matérielle d'un texte avec son intertexte, là où cette relation s'effectue dans les textes imprimés par un appareillage critique renvoyant à la consultation d'une source localisée dans un lieu distinct ${ }^{20}$.

$11 \mathrm{Au}$ sein des billets scientifiques de notre corpus, les fonctions assurées par l'enrichissement hypertextuel nous paraissent relever de quatre ordres: (i) éditorial, lorsqu'il sert la mise en lien de matériaux internes au carnet, par des renvois à des billets antérieurs ou par des procédés de redocumentarisation comme les rubriques ou les tags; (ii) critique, lorsqu'il permet la mise en relation du texte avec les sources primaires et secondaires de la recherche ; (iii) informatif, lorsque la délinéarisation du discours apporte un complément à même de favoriser la compréhension du texte et (iv) ludique, mobilisant des éléments de culture populaire à des fins de connivence avec le lectorat, qui rendent le propos plus léger et, dès lors, favorisent l'appréhension du savoir. Du point de vue de la cohérence, les textes liés assurant les fonctions (i) et (ii) sont susceptibles de créer, par rapport au texte du billet, un sentiment de textualité plus élevé que les deux derniers, dans le chef d'un public académique du moins: en effet, la fonction éditoriale situe la démarche de savoir dans une historicité propre, et l'activation de l'hyperlien en pôle réception offre à l'allocutaire la possibilité de reproduire le geste herméneutique du chercheur en donnant à voir l'interprétation du texte (Souchier et Jeanneret 1999, 106). Il serait toutefois un peu rapide d'inférer que l'enrichissement hypertextuel à fonction ludique ou informative crée forcément un sentiment de textualité moindre; ils nous semblent plutôt de nature à favoriser ce jugement chez un autre type de lectorat, le public élargi anticipé par la science ouverte $^{21}$ où les attendus du blog comme mode de communication informel suppose d'une part la possibilité de s'approprier des informations complémentaires en fonction de son socle de connaissances effectif (p. ex. par le renvoi vers des notices de l'encyclopédie Wikipédia : dans le billet $n^{\circ} \underline{78}$, les termes «Proxy » et «VPN » y trouvent une explicitation), et d'autre part de bénéficier d'un message agrémenté de clins d'œil (p. ex. dans le billet $\mathrm{n}^{\circ} \underline{18}$, où le technosegment final renvoie vers un sketch des Monty Pythons sur YouTube) ${ }^{22}$. 

le jugement de connexité : cet extrait du billet $n^{\circ} \underline{7}$ présente ainsi un liage du signifié où l'intertexte à valeur informative, matériellement présent sous la formes d'une URL embarquée dans le technomot, fait l'objet d'une désignation par le déictique ici :

Fig. 1: Extrait du billet $n^{\circ} 7$ (capturé le 18 décembre 2018)

La création d'un sentiment de connexité et de cohérence, en pôle production, a vocation à susciter par la manipulation de l'écrilecteur l'actualisation d'une formetexte, que l'on pourrait ainsi qualifier, à la suite d'Audet, de processuelle (Audet 2015) ${ }^{23}$. Cette manipulation n'a de chances d'advenir que si elle entre dans un plan d'usage qui correspond, ici, à l'acquisition de savoir sur un objet précis. De ce point de vue, on a signalé que deux types de publics étaient susceptibles d'être anticipés - pouvant d'ailleurs se recouper: rien n'empêche en réalité un universitaire d'être aussi un lecteur de blogs culinaires, journalistiques, etc., ou encore d'intervenir sur un blog scientifique ressortissant à une autre discipline que la sienne; les usages attendus répondent néanmoins à des motivations cognitives différentes. De ce fait, l'hyperlien constitue une adresse au lecteur, une figure de la lecture, pour reprendre l'idée de Saemmer (Saemmer 2015) - on voit d'ailleurs dans l'exemple cité que l'invite à la délinéarisation va de pair avec une interpellation directe du lecteur. L'imaginaire conversationnel du blog, reposant essentiellement sur la possibilité de réagir au billet par des commentaires, se trouve conforté par la présence de telles adresses. Peut-on considérer, dès lors, que le billet source fait texte avec le texte hyperlié ? En pôle réception, c'est une possibilité, suivant les choix de navigation du lecteur et ses usages. En pôle production, par contre, il ne nous semble pas que ce soit le cas, bien que l'URL embarqué fasse figure d'ajout et marque une saillance, un "état émergeant de la "forme-texte" » (Davallon et al. [2003b] 2013, paragr. 39) en attente d'actualisation.

Contrairement à l'activation d'hyperliens, le commentaire laisse une trace pérenne d'un acte d'écrilecture dans la matérialité du billet. Si l'écrilecteur apparaît bien comme un locuteur second, son discours s'inscrit dans les cadres énonciatifs du billet générés par l'architexte à la suite du texte premier ${ }^{24}$ et correspond aux attendus de ce format communicationnel; ce qui favorise un jugement de textualité sur l'ensemble ainsi constitué.

\section{Des commentaires inscrits dans l'imaginaire dialogique du blog}

Dacos et Mounier qualifiaient les carnets de recherche d'Hypothèses comme «espace[s] d'une conversation scientifique décentrée " (Dacos et Mounier 2010), renouant avec l'imaginaire du séminaire ou de la conférence académique; plus largement, le contexte de la science ouverte, visant l'affaiblissement des barrières d'accès aux textes savants, entretient l'imaginaire de la grande conversation scientifique (Guédon 2014). Le commentaire, constitutif de la forme-blog, s'associe dès lors à des enjeux de savoir particuliers que nous allons tenter de cerner. 

second produit dans un espace dédié scripturalement et énonciativement contraint au sein d'un écosystème numérique connecté » (Paveau 2017, 40). Elle en propose une typologie fonctionnelle: les commentaires peuvent être relationnels (établissant une relation phatique avec le texte premier, comme les like); conversationnels (qu'ils soient discursifs, échangeant sur le contenu, ou métadiscursifs, portant sur l'exercice de la pratique sociale liée à la scène d'énonciation [p. ex., dans les blogs de journalisme]) ou délocalisés (les commentaires figurent alors dans des échanges sur les messageries privées des réseaux sociaux). Ils peuvent encore être produits par partage, l'action même de faire circuler le contenu participant d'une forme de commentaire ${ }^{25}$. [2010] 2018), signalant par technosegment un lieu numérique citant le billet et établissant par-là une relation avec celui-ci (on en trouvera des exemples dans les billets $n^{\circ} \underline{14}, \underline{26}, \underline{45}, \underline{68}$, etc.). Les commentaires relationnels, phatiques, consistent en des remerciements (billets $n^{\circ} \underline{6}, \underline{26}$, etc.) ou félicitations (billet $n^{\circ} \underline{17}, \underline{78}$, etc.) ; auxquels on peut ajouter les multiples occurrences du commentaire-type signalant au carnetier la publication de son billet à la Une d'Hypothèses (p. ex. billet $\left.\mathrm{n}^{\circ} \underline{12}, \underline{84}\right)^{26}$. La cohérence avec le technodiscours premier du billet apparait dans ce cas plutôt faible, bien que la cohésion soit assurée par l'architexte qui affiche les commentaires dans des cadres énonciatifs liés au billet, sans cette fois qu'une action de l'écrilecteur ne nécessite l'actualisation de ces textes seconds. En revanche, cette cohérence s'intensifie dans le cas des commentaires discursifs et métadiscursifs, qui, dans le premier cas, rebondissent sur le billet en suggérant des ajouts ou des corrections et, dans le second cas, questionnent les attendus de la communication scientifique sur blog.

L'aspect conversationnel du blog s'illustre à travers des questions de contenu posées par les lecteurs aux carnetiers: demandes d'éclaircissements (billet $n^{\circ} \underline{78}$ ), de complément d'information factuel (billet $n^{\circ} \underline{42}$ ), ou questions méthodologiques liées à l'exploitation des ressources (billets $n^{\circ} \underline{11}, \underline{27}$ ). Le lecteur peut encore jouer un rôle de co-constructeur du savoir, par l'apport d'un complément d'information (billets $\mathrm{n}^{\circ} \underline{45}$, 87), de conseils de lectures et/ou de suggestions méthodologiques adressées au carnetier (p. ex. billets $n^{\circ} \underline{32}, \underline{41}, \underline{68}$ ), de sources non considérées (billet $n^{\circ} \underline{43}$ ); voire encore par la soumission d'hypothèses de recherche (billet $n^{\circ} \underline{68}$ ), ou l'ouverture d'un nouveau questionnement invitant à pousser plus loin la réflexion (billets $n^{\circ} \underline{44}, \underline{78}$ ). Il peut également se faire critique plus ou moins virulent, signalant des erreurs (p. ex. billet $n^{\circ} 63$ ) tout en invitant éventuellement le carnetier à un échange en vue d'une mise à jour du billet, qui est alors signalée par l'auteur en réponse au commentaire (billet $n^{\circ} \underline{39}$ ). Dans le même registre, un commentaire au billet $n^{\circ} \underline{43}$ y dénonce un possible parti-pris, qui ne tiendrait pas compte des acquis d'un ouvrage de référence objection à laquelle répond la carnetière.

19 Les aspects discursifs se mêlent, dans les commentaires des billets du corpus, aux aspects métadiscursifs, l'obtention de résultats de recherche étant inséparable d'une méthode de travail dans le champ scientifique (méthode qui est, par ailleurs, au fondement des disciplines). Pour Paveau, "[l]e commentaire est alors un lieu de renégociation des pratiques professionnelles et de légitimation professionnelle » (Paveau 2017, 47) : on voit ainsi comment les commentaires sont aussi un lieu où se dessine l'image du chercheur ${ }^{27}$ et où se négocie, le cas échéant, le périmètre de l'activité de recherche. Les commentaires du billet $n^{\circ} \underline{83}$ offrent une illustration remarquable de 
ce phénomène, portant non sur la pertinence du sujet abordé (la visite d'un musée en ex-RDA durant les vacances de la carnetière) mais sur la démarche de restitution de l'expérience dans un carnet de recherche (« est-on vraiment ici dans une démarche historique ?»). Le commentateur interroge ainsi la frontière de ce qu'autorise le carnet dans la communication d'expériences personnelles :

Fig. 2. Extrait du billet $n^{\circ} 83$ (capturé le 18 décembre 2018)

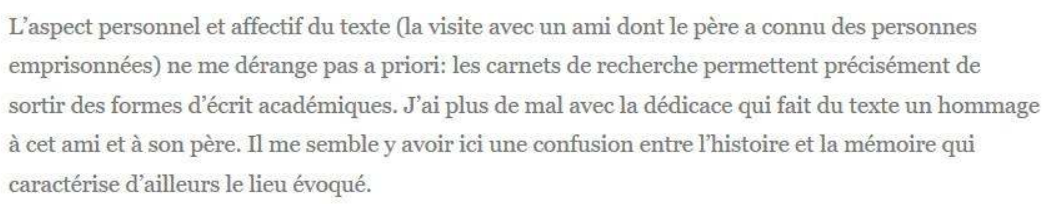

La carnetière répond ici aux critiques en jouant sur la multiplicité des casquettes ${ }^{28}$ :

Fig. 3. Extrait du billet $n^{\circ} 83$ (capturé le 18 décembre 2018)

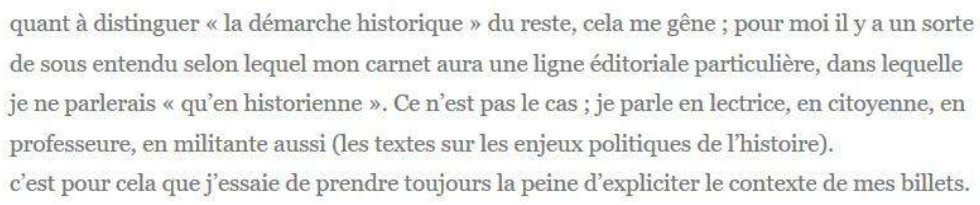

Enfin, certains commentaires n'appellent pas forcément réponse et sont davantage un espace d'expression investi par le lecteur, renvoyé à son propre vécu, ses propres pratiques de recherche. Il peut ainsi enregistrer et avaliser le contenu du billet, en ce qu'il en ratifie les observations (p. ex. billet $n^{\circ} \underline{85}$, sorte d'accusé de réception par l'allocutaire directe du billet épistolaire; billet $n^{\circ} \underline{80}$ témoignant de la réflexivité d'un chercheur qui avalise le propos du billet et s'engage à modifier ses propres pratiques : "Je vais essayer de changer mon comportement. »). Dans les commentaires du billet $\mathrm{n}^{\circ} \underline{\underline{3}}$, outre les félicitations dispensées sur le contenu du billet, un participant apporte un soutien à l'auteure face aux critiques qui lui sont faites. À nouveau, le sentiment de cohérence peut ici se fonder sur le rattachement du billet à l'imaginaire de la conversation scientifique, supposant la mise en commun des pratiques de recherche; il est d'autant plus fort que le chercheur-carnetier répond aux commentaires et entre en dialogue avec eux - voire, dans certains cas, dialogue avec lui-même, par exemple en signalant une mise à jour par un autocommentaire (billet $n^{\circ} \underline{54}$ ): dans ce cas précis, le commentaire se fait figure d'ajout comme « lieu d'un dédoublement réflexif de la figure de l'énonciateur » (Authier-Revuz et Lala 2002,10) qui opère alors un retour distancié sur une énonciation première. Le dispositif $\mathrm{du}$ blog, et plus particulièrement la fonctionnalité technogénérique du commentaire, est ainsi investi par l'usager (entendu ici comme le scripteur du carnet) non pas dans l'optique d'un dialogue avec autrui, qui serait celle attendue, mais dans celle d'une mise en visibilité des strates d'écritures, donnant davantage de corps à l'imaginaire du billet scientifique comme lieu d'une recherche en train de se faire. Dans son ouvrage sur la logique de l'usage, Perriault montrait en quoi les usages résultaient d'une négociation entre l'usager, le dispositif médiatique qu'il manipule et la visée communicationnelle souhaitée (Perriault 1989). En l'absence de fonctionnalité spécifiquement dédiée au signalement des mises à jour du contenu des billets sur Hypothèses, l'espace du commentaire se trouve détourné de 
son usage premier pour donner à voir un ajout autographe mentionnant un apport informationnel.

D'une manière analogue, les commentaires délocalisés du billet $n^{\circ} \underline{51}$ recourent à la fonctionnalité de citation de l'architexte WordPress pour mettre en évidence un dialogue de la carnetière avec d'autres usagers, amorcé dans les commentaires d'un billet antérieur - en effet, contrairement à ce que l'on pourrait trouver dans un forum de discussion, la fonction "répondre » d'Hypothèses ne prévoit pas la reprise partielle d'un contenu en vue d'une réponse ciblée. Le texte est écrit à la suite des discussions suscitées par un précédent billet ${ }^{29}$, recension d'un spectacle intitulé Le Tartuffe inconnu de Molière et joué alors à la Sorbonne par des étudiants et anciens étudiants. La pièce, appuyée sur l'étude de sources historiques, tentait de restituer la pièce originelle avant ses multiples censures et réécritures, telle qu'elle avait pu être représentée dans sa version initiale aux Plaisirs de l'île enchantée $e^{30}$. La carnetière évalue dans un premier billet les réussites et points faibles d'une telle adaptation, ce qui entraîne entre autres la réaction d'un spectateur partageant partiellement ses opinions, ainsi que du chercheur ayant supervisé la (re)création de la pièce qui apporte de longues précisions quant aux options scénaristiques et scénographiques retenues. Le billet $\mathrm{n}^{\circ} \underline{51}$ vise alors à apporter une réponse à ce long commentaire, dont des extraits sont reproduits comme autant de blocs citationnels restituant le point de vue du chercheur, et permettant à la carnetière de dialoguer avec lui point par point (dialogue qui se poursuit, à nouveau, dans les commentaires du billet $n^{\circ} \underline{51}$; ce que n'autorisent pas les fonctionnalités du blog WordPress où la réponse à un commentaire ne peut porter que sur l'ensemble de celui-ci. On voit ainsi de quelle manière les carnetiers d'Hypothèses font preuve de créativité dans l'investissement du dispositif technique et détournent les fonctionnalités existantes pour répondre au mieux à leurs besoins.

On ajoutera enfin que, dans ce dernier cas de commentaire délocalisé au sein même d'un nouveau billet, le jugement de cohérence sur l'unité textuelle corps du billetcommentaire est susceptible d'être plus fort encore que dans les autres types de commentaires évoqués. Se pose la question du texte à considérer : ne dépasse-t-il pas ici les frontières du billet, et ne devrait-on pas dès lors envisager le carnet comme texte, jugement qui serait ici renforcé par les technomots à fonction éditoriale des deux premiers paragraphes, renvoyant vers des billets antérieurs?

\section{Conclusion : partage et discussion, des gestes discursifs du savoir participant de la textualisation du discours scientifique}

L'analyse du corpus montre que les billets résultent d'actes énonciatifs complexes, émanant d'acteurs divers (locuteur, écrilecteur, concepteurs de l'architexte, etc.). L'architexte favorise le sentiment de cohésion, en ce qu'il est à l'origine des cadres textuels organisant les contenus du blog et permet l'embarquement de données et métadonnées en vue de la circulation du texte dans des espaces externes comme les réseaux sociaux. Le jugement de cohérence se joue à travers l'évaluation de l'adéquation du texte avec les attendus de l'hypergenre du blog (dont participent tant les technomots menant vers des écrits hyperliés que les commentaires) ainsi qu'avec la scène d'énonciation ${ }^{31}$ scientifique où s'inscrit Hypothèses: à cet égard, l'enrichissement hypertextuel à fonction critique et les commentaires assurant une fonction 
conversationnelle sont sans doute les plus à même de renforcer le jugement de cohérence thématique. Le jugement de textualité se trouve conforté, le cas échéant, par la présence de liage micro-textuels qui favorisent le sentiment de connexité. Si l'on n'en a pas dressé l'inventaire et que l'on s'est bornée à signaler un exemple de liage du signifié, un autre type de liage des unités discursive est assuré, selon Adam, par les actes de discours: il signale ainsi qu' " $[u] n$ texte n'est pas une simple suite d'actes d'énonciation possédant une certaine valeur ou force illocutoire [...], mais une structure d'actes de discours liés" (Adam 2011, 154). Il nous semble que l'enrichissement hypertextuel du discours scientifique, tout comme le commentaire, ont quelque chose à voir avec ces actes de discours. Plus précisément, on pourrait les rattacher à ce que le collectif liégeois LTTR13 désigne comme gestes discursifs du savoir. La notion y est envisagée comme un pendant de la figure de style, agissant sur le plan de l'énonciation :

La conception du geste discursif active cette distinction proposée aux marges de la rhétorique [...] entre le figuratif et le figural. Tandis que la figure possède un versant figuratif très affirmé, le figural donne forme à la pensée et montre de quelle pensée le geste est le soutien. Le geste discursif n'est en effet pas nettement circonscriptible dans l'énoncé, mais laisse saisir à travers des traces la manière figurale dont un discours progresse. (Lttr13 2016, 94-95)

Définis comme saillance[s] de l'énonciation (Ibid., 97), les gestes discursifs du savoir sont situés par LTTR13 au regard de deux concepts linguistiques : (i) les séquences textuelles d'Adam comme modules compositionnels (Adam 2011): les gestes discursifs correspondraient à de telles séquences, sur le plan de l'énonciation; (ii) les actes de langage, qui dotent les énoncés d'une force illocutoire: les gestes discursifs seraient alors à considérer en tant qu'actes énonciatifs. Les gestes discursifs du savoir consigneraient ainsi la trace des conditions de production des connaissances en manifestant, sous l'elocutio, qui apparaît comme l'aboutissement rhétorique du discours, les étapes préparatoires que sont l'inventio (l'inventaire des arguments mobilisables) ou la dispositio (leur organisation à des fins d'efficacité argumentative) (Ibid., 98). Problématisation, questionnement, exhibition des pratiques appartiennent, par exemple, à la palette des gestes discursifs du savoir. Ces gestes ne sont pas des modules isolés, mais apparaissent liés en ce qu'ils organisent la progression thématique des opérations de construction de la connaissance et participent d'une dramatisation dans la communication de la recherche.

Si l'on s'accorde à suivre cette perspective, loin de mettre à mal l'unité textuelle, technomots et commentaires pourraient au contraire favoriser le jugement de textualité sur le discours scientifique du billet, en ce que le sentiment de connexité dépendrait, entre autres, des gestes discursifs du savoir opérant un liage d'unités textuelles. Le commentaire correspondrait ainsi à un geste discursif du savoir du type discussion, tandis que l'enrichissement hypertextuel manifesterait un partage ${ }^{32}$ de ressources que l'écrilecteur choisirait ou non d'actualiser selon ses motivations cognitives. Il faut toutefois signaler que le blog scientifique d'Hypothèses ne saurait être le lieu d'une elocutio définitive en ce que, d'une part, inscrit dans l'imaginaire du carnet de recherche, il est pensé comme un espace de première consignation d'idées et d'observations n'ayant pas encore trouvé une forme aboutie; et que, d'autre part, la possibilité d'interagir par les commentaires fait du billet un texte toujours ouvert, susceptible d'agrégations sur le temps long: de ce point de vue, le commentaire ne 
manifesterait pas l'inventio ou la dispositio sous-jacente, mais davantage une disputatio qui prolongerait l'elocutio - ce que signalaient déjà Dacos et Mounier (Dacos et Mounier 2010 , 9). Par ailleurs, résultant d'une énonciation seconde liée architextuellement au discours premier, le commentaire est nettement circonscrit dans un cadre visuel, là où les gestes discursifs du savoir sont conçus comme des saillances énonciatives manifestant un mouvement de pensée qu'il est difficile de borner de manière précise. L'étude des technomots et commentaires comme manifestations d'une trace de la pensée en train de s'élaborer, que rendent saillantes des dispositifs informatisés comme le blog, permettraient d'ouvrir la réflexion sur de nouveaux gestes discursifs du savoir, intégrant la multiplicité des instances énonciatives à l'œuvre dans les textes scientifiques numériques.

\section{BIBLIOGRAPHIE}

Tous les liens hypertextes du document ont été vérifiés le 28 décembre 2018.

Adam, Jean-Michel (2011). La linguistique textuelle : Introduction à l'analyse textuelle des discours, Paris : Armand Colin.

Adam, Jean-Michel (2015). Faire texte : frontières textuelles et opérations de textualisation, Besançon : Presses universitaires de Franche-Comté.

Audet, René (2015). « Écrire numérique : du texte littéraire entendu comme processus ». Itinéraires. Littérature, textes, cultures, https://doi.org/10.4000/itineraires.2267

Authier-Revuz, Jacqueline, et Marie-Christine Lala, éd. (2002). Figures d'ajout : phrase, texte, écriture, Paris : Presses Sorbonne Nouvelle.

Barbosa, Pedro (1992). Metamorfoses do real. Criaçâo literaria e computador, Lisbonne : Universidade nova de Lisboa.

Beaudry, Guylaine (2011). La communication scientifique et le numérique, Paris : Lavoisier.

Candel, Étienne (2010). «Penser la forme des blogs, entre générique et génétique ». Itinéraires. Littérature, textes, cultures, $\mathrm{n}^{\circ}$ 2010-2 (juillet) : 23-31. https://doi.org/10.4000/itineraires.1932

Combe, Dominique (2002). «L'ajout en rhétorique et en poétique », In Authier-Revuz Jacqueline et Marie-Christine Lala (éds), Figures d'ajout : phrase, texte, écriture, Paris : Presses Sorbonne Nouvelle, 15-27.

Commission Européenne, éd. (2016). Open Innovation, Open Science, Open to the World: A Vision for Europe, Luxembourg: Publications Office of the European Union.

Couleau, Christèle, et Pascale Hellégouarc'h, éd. (2010). « Les blogs. Écritures d'un nouveau genre? » Itinéraires. Littérature, textes, cultures, https://journals.openedition.org/itineraires/ $\underline{1916}$

Dacos, Marin, et Pierre Mounier (2010). « Les carnets de recherche en ligne, espace d'une conversation scientifique décentrée », In Jacob Christian (éd.), Lieux de savoir. 2. Gestes et 
supports du travail savant, Paris : Albin Michel, http://archivesic.ccsd.cnrs.fr/sic_00439849/ document

Davallon, Jean, Marie Després-Lonnet, Yves Jeanneret, Joëlle Le Marec, et Emmanuël Souchier (2013 [2003a]). Lire, écrire, récrire : Objets, signes et pratiques des médias informatisés, Paris : Éditions de la Bibliothèque publique d'information, http://books.openedition.org/bibpompidou/ $\underline{394}$

Davallon, Jean, Marie Després-Lonnet, Yves Jeanneret, Joëlle Le Marec, et Emmanuël Souchier (2013 [2003b]). « Conclusion », In Lire, écrire, récrire : Objets, signes et pratiques des médias informatisés, Paris : Éditions de la Bibliothèque publique d'information, 303-22, http:// books.openedition.org/bibpompidou/424

Davallon, Jean, Nathalie Noël-Cadet, et Danièle Brochu (2013 [2003]). « Chapitre I. L'usage dans le texte : les "traces d'usage” du site Gallica », In Davallon, Jean, Marie Després-Lonnet, Yves Jeanneret, Joëlle Le Marec, et Emmanuël Souchier, Lire, écrire, récrire : Objets, signes et pratiques des médias informatisés, Paris : Éditions de la Bibliothèque publique d'information, 47-90, http:// books.openedition.org/bibpompidou/409

Détrie, Catherine (2008). « Textualisation et (re)conditionnement énonciatif / Textualisation and (re)formating of the relationship between the writer and the reader ».CD-Rom et archive électronique, juillet, 1319-32.

Ducrot, Oswald, et Tzvetan Todorov (1972). Dictionnaire encyclopédique des sciences du langage, Paris : Éditions du Seuil.

Gallezot, Gabriel (2016). « Publicisations, lettrures scientifiques et évolutions des modes éditoriaux » Conférence invitée, Université de Meiji Gakuin, Centre de Recherches Gengobunka (Tokyo), 28 septembre 2016, https://archivesic.ccsd.cnrs.fr/sic_01438208

Grossmann, Francis, et Laurence Rosier (2018). « Du discours rapporté au discours partagé. Analyser les usages du discours rapporté hypertextualisé ", In Simon Justine (ed.), Le discours hypertextualisé. Espaces énonciatifs mosaïques, Besançon : Presses universitaires de FrancheComté, 41-64.

Guédon, Jean-Claude (2014). « Chapitre 7. Le libre accès et la "Grande Conversation" scientifique », In Vitali-Rosati Marcello et Michael E. Sinatra (eds), Pratiques de l'édition numérique, Montréal : Presses de l'Université de Montréal, 111-26, http:// books.openedition.org/pum/324

Jahjah, Marc (2017). « “Annoter le monde et améliorer l'humanité”: les imaginaires matériels d'un logiciel d'annotation du web », In Gérald Kembellec et Evelyne Broudoux (éds), Écrilecture augmentée dans les communautés scientifiques : Humanités numériques et construction des savoirs, Paris : ISTE Éditions, 71-88.

Jeanneret, Yves (2005). « La culture triviale », Entretien réalisé par Équipe Sémiotique Cognitive et Nouveaux Médias. Archives Audiovisuelles de la Recherche. https://www.canal-u.tv/video/ fmsh/la_culture_triviale.28717

Jeanneret, Yves (2014). Critique de la trivialité : Les médiations de la communication, enjeu de pouvoir, Paris : Éditions Non Standard.

Jeanneret, Yves, et Emmanuël Souchier (2005). «L'énonciation éditoriale dans les écrits d'écran », Communication et langages 145 (1), 3-15, https://doi.org/10.3406/colan.2005.3351

Kembellec, Gérald, et Evelyne Broudoux, éd. (2017). Écrilecture augmentée dans les communautés scientifiques : Humanités numériques et construction des savoirs, Paris : ISTE Éditions. 
Latour, Bruno (1991). Nous n'avons jamais été modernes: essai d'anthropologie symétrique, Paris : La Découverte.

Le Marec, Joëlle (2002). « Situations de communication dans la pratique de recherche : du terrain aux composites ", Études de communication. Langages, information, médiations, $n^{\circ} 25,15-40$, https://doi.org/10.4000/edc. 831

Lttr13 (2016). «Figures de l'énonciation : les gestes discursifs du savoir », In Amir Biglari et Geneviève Salvan (éds), Figures en discours, Louvain-la-Neuve : Academia - L'Harmattan, 93-116, https://orbi.uliege.be/handle/2268/194724

Maingueneau, Dominique (2004). Le discours littéraire : Paratopie et scène d'énonciation, Paris : Armand Colin.

Maingueneau, Dominique (2013). «Genres de discours et web : existe-t-il des genres web ?» In Barats Christine (coord.), Manuel d'analyse du web en Sciences Humaines et Sociales, Paris : Armand Colin, 74-93.

Mayeur, Ingrid (2018). « Médiations informatisées et discours de savoir. Le blogging scientifique en SHS, un genre en construction ", Cahiers de praxématique, $n^{\circ} 71$, http://

journals.openedition.org/praxematique/5186

Mounier, Pierre, et Marin Dacos (2010). L'édition électronique, Paris : La Découverte.

Odin, Roger (2011). Les espaces de communication : introduction à la sémio-pragmatique, SaintMartin-d'Hères : Presses Universitaires de Grenoble.

Pacaud, François (2018 [2010]). « Pings et rétroliens, des liens réciproques entre billets », La Maison des carnets (blog), https://maisondescarnets.hypotheses.org/38

Paveau, Marie-Anne (2015). «Ce qui s'écrit dans les univers numériques ». Itinéraires. Littérature, textes, cultures, $\mathrm{n}^{\circ} 2014-1$, https://doi.org/10.4000/itineraires.2313

Paveau, Marie-Anne (2016). « Des discours et des liens. Hypertextualité, technodiscursivité, écrilecture », Semen - Revue de sémio-linguistique des textes et discours, $n^{\circ} 42,23-48$.

Paveau, Marie-Anne (2017). L'analyse du discours numérique. Dictionnaire des formes et des pratiques, Paris : Hermann.

Perriault, Jacques. 1989. La logique de l'usage : essai sur les machines à communiquer, Paris : Flammarion.

Rabatel, Alain (2017). Pour une lecture linguistique et critique des médias : Empathie, éthique, point(s) de vue, Limoges :Lambert-Lucas.

Rentier, Bernard (2018). Science ouverte, le défi de la transparence, Académie royale de Belgique, L'Académie en poche, Bruxelles.

Rinck, Fanny (2010). «L'analyse linguistique des enjeux de connaissance dans le discours scientifique », Revue d'anthropologie des connaissances 4 (3), 427-50.

Saemmer, Alexandra (2015). Rhétorique du texte numérique : figures de la lecture, anticipations de pratiques, Villeurbanne: Presses de l'Enssib.

Simon, Justine (2017). «Présentation [Le discours hypertextualisé] ». Semen. Revue de sémiolinguistique des textes et discours, $\mathrm{n}^{\circ} 42$, http://semen.revues.org/10607

Souchier, Emmanuël (2012). « La "lettrure” à l'écran », Communication \& langages, nº174, 8-108. https://doi.org/10.4074/S0336150012014068 
Souchier, Emmanuël, et Yves Jeanneret (1999). « Pour une poétique de «l'écrit d'écran» », Xoana, $\mathrm{n}^{\circ} 6 / 7,97-107$.

Vitali-Rosati, Marcello (2016). « Qu'est-ce que l'éditorialisation?» Sens Public, http://www.senspublic.org/article1184.html

\section{ANNEXES}

Table des illustrations

Fig. 1 : Troch, Kevin. 2016. «Exploitation minière et sismicité induite? Un éclairage historique sur une controverse d'actualité: l'exemple de la Belgique et du Nord de la France, années 1880-années 1980 (1/3)». Billet. Histoire de trous. Carnet de recherche en histoire environnementale minière (blog). 4 novembre 2016 [mise à jour du 17 février 2017]. https://trous.hypotheses.org/31. [extrait]

Fig. 2 et 3 : Muller, Caroline. 2018. « Mémoire de l'enfermement - la prison de la Stasi à Potsdam ». Billet. Acquis de conscience. Histoire(s) de direction de conscience au XIXe siècle (blog). 5 janvier 2018 [mise à jour du 9 mars 2018]. https:// consciences.hypotheses.org/1100. [extraits]

\section{NOTES}

1. Je tiens à remercier OpenEdition pour la communication des données nécessaires à la constitution de mon corpus, ainsi que Fanny Rinck et Étienne Candel, relecteurs de cet article, pour leurs remarques critiques ayant contribué à l'améliorer.

2. Le discours de savoir est ici entendu comme un discours par lequel on construit un savoir sur un objet, ce savoir étant susceptible d'être enseigné, transmis. Le discours de savoir concerne donc bien entendu les disciplines scientifiques, mais également le sport, l'art (culinaire, pictural, etc.). Nous remercions Sémir Badir pour les indications qu'il a bien voulu nous communiquer à ce sujet.

3. Il faut cependant signaler les propositions programmatiques de Dacos et Mounier dans leur ouvrage L'édition numérique (Mounier et Dacos 2010). Bien qu'elles n'interrogent pas fondamentalement le faire texte ni le discours scientifique, elles définissent pour le texte numérique l'articulation des trois critères que sont la lisibilité, la manipulabilité et la citabilité.

4. On renvoie ici aux travaux de Paveau en analyse du discours numérique, où le terme technodiscours signale le caractère indissociable, dans les discours numériques, des éléments langagiers et techniques: "parler de technodiscours, [c'est] affirmer que les discours numériques natifs ne sont pas d'ordre purement langagier, que les déterminations techniques coconstruisent les formes technolangagières, que les perspectives logo- et anthropocentrées doivent être écartées au profit d'une conception écologique intégrative qui reconnaisse le rôle des agents non humains dans les productions langagières. » (Paveau 2017, 11).

5. On a conscience que la qualification d'enrichissement hypertextuel peut être contestable, en ce que les énoncés numériques, comme composites (Paveau 2017), lient de manière indissociable la linguistique et le technique. "Enrichir» pourrait manifester une primauté de l'imprimé par rapport auquel le texte numérique ne serait qu'augmenté. On conservera toutefois la dénomination, moyennant la prise en compte de cette remarque, étant donné que nous proposons de lire les technomots/technosegments comme figures d'ajout, soit qui manifestent une rupture dans la temporalité première de l'énonciation (Authier-Revuz et Lala 2002, 10) - figure 
étant entendu, dans la perspective des éditrices du collectif Figures d'ajout, comme disposition, configuration (Ibid, 8). Au demeurant, les scripteurs de billets scientifiques sur Hypothèses ne créent pas d'énoncés directement relationnels (ce qui pourrait être le cas, par exemple, dans une conversation sur Twitter) mais insèrent le lien en sélectionnant un mot préalablement écrit via l'activation, dans la console de rédaction, de l'icône ad hoc (figurant, comme dans la plupart des architextes informatisés, deux maillons enchâssés).

6. Si le texte scientifique révisé par les pairs (comme l'article ou la monographie) porte également la trace de plusieurs contributeurs et apparaît ainsi comme le résultat d'un processus de co-construction du savoir, ces traces d'ajout ne sont généralement plus visibles au sein du texte final. Il existe toutefois des infrastructures de communication scientifique (plutôt dans le domaine des sciences dites "dures») où ces révisions restent apparentes, comme dans les articles du Self Journal of Science de Michaël Bon; on mentionnera aussi que, dans la présente livraison, Marcello Vitali-Rosati donne accès aux différentes strates d'écritures de son article par un lien hypertexte pointant vers son espace de travail.

7. Convoquant un discours autre qui, s'il est ainsi représenté, ne fait pas l'objet de paroles rapportées : voir à ce sujet les observations de (Rabatel 2017, 379).

8. Paveau ouvrait ainsi la porte à une lecture du discours hypertextualisé comme une forme d'« hétérogénéité techno-énonciative " (Paveau 2016, paragr. 51).

9. Sur l'ajout autographe/allographe, voir (Combe 2002, 17).

10. On pourrait établir un parallèle entre cette proposition d'Adam et la démarche sémiopragmatique d'Odin qui, à partir de la notion d'espace de communication, élabore des outils visant à étudier la manière dont le contexte règle l'activité de production du sens des textes (au sens large, incluant par exemple les productions filmiques). Dans ce cadre, "il ne s'agit plus d'analyser un texte existant, mais d'analyser l'expérience d'un travail de production textuelle en contexte » (Odin 2011, 123) ; soit, en quelque sorte, d'étudier comment s'exerce le jugement au regard de critères qui font sens dans un contexte donné.

11. En ce que les dispositifs médiatiques suggèrent, par leur matérialité même, des pratiques d'écriture; la suggestion étant entendue comme un "processus technique qui consiste à placer sous les doigts et sous les yeux de l'usager d'un média une conception de la communication, plutôt que de l'expliquer par le langage » (Jeanneret 2014, 15).

12. Sur le genre du blog, on consultera utilement la livraison de la revue Itinéraires intitulée «Les blogs, écritures d'un nouveau genre? » (Couleau et Hellégouarc'h 2010). Sur le blog de recherche comme genre du discours scientifique, nous renvoyons à notre contribution (Mayeur 2018).

13. On utilisera ici le terme dans un sens éditorial, soit celui d'une inscription dans le réseau et d'une valorisation des contenus sur le web. Au sens large, Vitali-Rosati définit l'éditorialisation comme «l'ensemble des dynamiques qui produisent et structurent l'espace numérique. Ces dynamiques sont les interactions des actions individuelles et collectives avec un environnement numérique particulier. » (Vitali-Rosati 2016).

14. On signalera que le champ de la praxématique a également étudié la dimension interactionnelle de la textualité, locuteur et allocutaire étant engagés en tant que coénonciateurs dans le processus de textualisation (voir par exemple la mise au point dans Détrie 2008).

15. La littéracie a, elle aussi, affaire avec la lettrure, en tant qu'elle englobe les pratiques d'écriture et de lecture dans l'accès aux savoirs - ainsi que le rappelle Souchier (Souchier 2012, § 7). Ces savoir-faire héritent pour une part de la culture imprimée, mais ont évolué du fait de la fréquentation des écrits d'écran.

16. On reprend ici un terme de Davallon et al., pour qui cette négociation «implique une proposition textuelle du site et une coopération de l'usager » (Davallon, Noël-Cadet, et Brochu 2013, § 100). 
17. Ces billets ont fait l'objet d'une sélection et d'une valorisation émanant du comité éditorial de la plateforme ; ils semblent de ce fait recueillir davantage de commentaires que la moyenne. Une description plus fine de ce corpus est consultable dans une bibliothèque publique Zotero (https://www.zotero.org/groups/2218907/corpus_thse_ingrid_mayeur/items, dossier «Corpus $1 »)$; une liste bibliographique est également disponible sur notre carnet de recherche (https:// driv.hypotheses.org/corpus-de-these). Par souci de fluidité, on a choisi de recourir à l'enrichissement hypertextuel dans le corps de l'article. En cas de corruption des liens, les captures restent toutefois documentées au sein du dépôt Orbi, à partir de la notice associée à notre travail doctoral.

18. Nous nous inspirons ici de la dénomination discours hypertextualisé, que l'on doit à Simon (Simon 2017).

19. Marcoccia observait ainsi que les écrits numériques «manifestent un haut degré d'intertextualité, par la présence (plus ou moins littérale ou intégrale) de textes dans d'autres textes.» (Marcoccia 2016, 100). Il rapporte toutefois que Vandendorpe distinguait entre l'intertexte comme fait de lecture, et l'hypertexte comme construit informatique (Vandendorpe 1999; cité par Marcoccia 2016, 105). Paveau invite encore, pour sa part, à ne pas confondre relationalité et dialogisme, la relationalité des discours numériques étant matérielle et automatique (Paveau 2017, 13).

20. Grossmann et Rosier indiquent ainsi que la mise en relation hypertextuelle permet «la coexistence matérielle, synchronique de l'énoncé premier et de l'énoncé ajouté. » (Grossmann et Rosier 2018, 45). On doit à ces auteurs une première investigation sur le rôle des liens hypertexte dans les discours de savoir, intégrant certains billets d'Hypothèses essentiellement envisagés, au demeurant, sous la forme de vignettes défilant sur la page d'accueil de la plateforme (Ibid.).

21. Inscrite dans le sillage du mouvement de l'accès ouvert, visant l'abolition des barrières payantes aux textes scientifique, la science ouverte préconise également l'accès aux données de la recherche (autorisant, par exemple, les pratiques de text and data mining), et l'utilisation de logiciels non propriétaires. Elle promeut une démarche scientifique collaborative et une large diffusion des connaissances, impliquant le cas échéant un public extra-académique qui se trouvent également sollicités dans la démarche de construction des savoirs (citizen science). Voir à ce sujet (Commission Européenne 2016 ; Rentier 2018).

22. Dans ce dernier cas, la cohérence thématique est également mise à mal : il y a ainsi une démarche consistant à déjouer les attentes de l'allocutaire au regard de la scène d'énonciation considérée, celle d'une plateforme de communication de la recherche.

23. "Ainsi, le texte numérique se définit par le processus qui le voit naître bien davantage que par son état.» (Audet 2015, § 10); l'auteur désigne alors plus spécifiquement les opérations d'écriture dans les œuvres littéraires. Cette processualité repose sur quatre composantes: négocier une contrainte médiatique (ce dont on rend compte ici en recourant au concept d'architexte issu des SIC), se réticuler (par la mise en relation hypertextuelle), fluctuer (par le jeu des possibles qu'entraîne l'ouverture d'un texte inscrit dans un flux) et s'épandre (l'ouverture du texte allant de pair avec des possibilités d'extensions inédites).

24. Contrairement, par exemple, à un logiciel d'annotation comme Hypothes.is, lié au navigateur, qui dès lors apparaît comme externe à l'architexte. Sur ce logiciel et ses usages, voir (Jahjah 2017).

25. Sans entrer ici dans les détails, on notera que, dans la perspective de Paveau, le partage est lié aux phénomènes de technodiscours rapportés. Ceux-ci consistent à «transférer un discours d'un espace numérique natif source à un espace numérique natif cible, via une procédure automatisée de partage » (Paveau 2017, 289). Ce type de partage peut s'effectuer suivant différentes modalités morphologiques: Paveau distingue ainsi entre technodiscours rapporté intégral, résumant et répétant: si les deux premiers types concernent une réplication totale ou partielle du contenu accompagnée ou non d'un commentaire, le dernier reproduit à l'identique le discours cité. 
26. Celui-ci est libellé comme suit: "Chère carnetière, cher carnetier, Nous avons particulièrement apprécié votre billet. Pour que la communauté puisse plus aisément le découvrir, nous avons décidé de le mettre en Une d'Hypothèses. Bien cordialement, L'équipe d'Hypotheses.org ".

27. Ce qui tend à situer le commentaire sur blog dans une continuité des pratiques scientifiques, puisque ce positionnement discursif se joue d'une manière tout à fait similaire dans les commentaires des conférences.

28. Les commentaires de blogs étant souvent un espace polémique, le premier commentateur se soucie vite de faire baisser la tension et de resituer sa réflexion dans le cadre d'une intervention sur les contours de la scène d'énonciation scientifique sur la plateforme Hypothèses : «Je ne vous accuse surtout pas ! Je suis un fidèle lecteur et j'apprécie votre démarche. Je suis embêté que vous donniez votre légitimité d'historienne et de carnetière à ce Mémorial qui me pose problème. D'autant plus embêté que ce billet est à la une. Mais les lectrices et lecteurs qui viendront en nombre ici participeront peut-être à ce débat pour nous aider à y voir plus clair.» (billet $n^{\circ} \underline{83}$ ).

29. Roussillon, Marine. 2017. "Tartuffe : retour aux origines". Billet. Politiques du Grand-siècle (blog). 25 avril 2017. https://pogs.hypotheses.org/338.

30. L'expression désigne les festivités organisées par Louis XIV à Versailles en mai 1664.

31. Sur la scène d'énonciation comme cadre et processus de l'activité de parole: voir (Maingueneau 2004).

32. Pour Grossmann et Rosier, le discours hypertextuel assumerait des fonctions pragmatiques et évidentielles par rapport au discours source, le locuteur se positionnant par l'acte même de la citation numérique comme interlocuteur à même de faire circuler les discours dans cet environnement. Ils notent ainsi que les textes ne sont plus tant rapportés que partagés, « fournis en arrière-plan, créant ainsi un horizon d'attente générateur d'hypothèses de lectures, et entrant aussi dans un système de validation. » (Ibid., 50). Le partage est donc ici entendu comme un effet pragmatique associé à l'usage du discours hypertextualisé ; sens dans lequel nous utilisons ici le terme. Dans la conception de Paveau, on a vu que le partage relève en revanche, comme le commentaire, d'une énonciation seconde greffée sur un texte premier et se matérialiserait donc dans un espace autre - ce que nous n'étudions pas ici pour les billets d'Hypothèses, même si l'on en a fait mention.

\section{RÉSUMÉS}

Le présent article propose des pistes pour déterminer par quels moyens le discours scientifique sur blog fait texte. Nous nous intéressons plus spécifiquement à l'enrichissement hypertextuel des énoncés et aux commentaires qui interrogent les contours du billet scientifique comme forme textuelle cohérente. À partir de l'analyse d'un corpus de 87 billets extraits de la Une d'Hypothèses, plateforme de carnets de recherche en sciences humaines et sociales, nous identifions les fonctions cognitives assurées par ces deux composantes des écrits de blogs scientifiques ainsi que leur incidence sur le jugement de textualité (Adam 2011) susceptible d'être porté sur les billets. Nous proposons enfin une lecture de l'enrichissement hypertextuel et des commentaires comme gestes discursifs du savoir (Lttr13 2016) jouant un rôle dans les opérations de textualisation des billets scientifiques. 
This article proposes some lines to determine how scientific discourses on blogs are likely to make text. It focuses more specifically on hypertextual enrichment and comments that question textual boundaries. The analysis based on a corpus of 87 posts extracted from Hypothèses ( $\mathrm{a}$ platform of blogs in the Humanities) identify the cognitive functions provided by these two components and their impact on the textual judgment (Adam 2011) that may be carried on the posts. Finally, it proposes a reading of hypertextual enrichment and comments as discursive gestures of knowledge (Lttr13 2016) that play a role in the textualization process of scientific posts.

\section{INDEX}

Mots-clés : Gestes discursifs de savoir ; discours scientifique ; discours numérique ; blogging scientifique ; commentaires ; textiel ; hypertextualité numérique ; technodiscours

Keywords : Discursive gestures of knowledge; scientific discourse; digital discourse; scientific blogging; comments; digital hypertextuality ; technodiscourse

\section{AUTEUR}

\section{INGRID MAYEUR}

Université de Liège (Belgique) 